This is the post-print version of this article: Meeus, L., Vandezande, L., Cole, S., Belmans, R., 2009. Market coupling and the importance of price coordination between power exchanges. Energy, 34(3), pp 228-234.

\title{
Market Coupling and the importance of price coordination between power exchanges
}

\author{
L. Meeus ${ }^{*}$, L. Vandezande ${ }^{1}$, S. Cole ${ }^{2}$, R. Belmans ${ }^{3}$ \\ Electrical Engineering Branch, Energy Institute, University of Leuven (KUL) \\ Kasteelpark Arenberg 10 / B-3001 Leuven / Belgium
}

\begin{abstract}
In Europe, market coupling stands for a further integration of wholesale trading arrangements across country borders. More specifically, it refers to the implicit auctioning of cross-border physical transmission rights via the hourly auctions for electric energy organized by power exchanges one day ahead of delivery. It therefore implies that the power exchanges can optimize the clearing of their day-ahead auctions. Due to verticals in the aggregated order curves, the optimal solution can be settled at different

\footnotetext{
* Correspondence to: Leonardo Meeus, Electrical Engineering Department ESAT, University of Leuven (KUL), Kasteelpark Arenberg 10, 3001 Leuven, Belgium; E-mail: leonardo.meeus@esat.kuleuven.be; Fax: ++32-(0)16/321985.

${ }^{1}$ E-mail: leen.vandezande@esat.kuleuven.be

${ }^{2}$ E-mail: stijn.cole@esat.kuleuven.be

${ }^{3}$ E-mail: ronnie.belmans@esat.kuleuven.be
} 
prices. In order for prices to give correct signals for network development and where to locate generation and consumption, price coordination between exchanges is necessary. The paper illustrates this issue, its relevance and discusses how to deal with it.

KEY WORDS: Pricing, market equilibrium, operations research, power system economics, and duality

\section{INTRODUCTION}

In Europe, generators self-schedule and they do this by submitting a program to the network operator. When which generators are turned on and run is the result of trade in several types of markets. Trade is mainly bilateral, but in most countries this is supplemented with auctions organized by power exchanges one day ahead of delivery for every hour of the next day. The auctions are used by market parties to fine tune their portfolios, which for instance means that generators can be on the supply as well as demand side depending on whether they are long or short. The exchanges use simple rules to settle contracts one day ahead of delivery when it is not worth getting into time consuming bilateral negotiations. Additionally, the exchanges act as counter-party for all transactions. The traded volume on the exchanges is typically only $10 \%$ of consumption ${ }^{4}$.

\footnotetext{
${ }^{4}$ Exceptions are for instance Nord Pool and Omel, respectively the Scandinavian and Spanish exchange who have a traded volume close to $100 \%$ of consumption, but this is at least partly due to liquidity
} 
While wholesale trade within countries is not constrained by the network, it is constrained at the borders where there are structural bottlenecks. The Transmission System Operators (TSOs) determine transfer capacities (so-called Net Transfer Capacities) independently per border and before trade actually takes place. In other words, before it is known how flows will be distributed over the different border lines and without taking the interdependencies of a meshed network into account. About $10 \%$ of consumption is traded across borders in Europe.

As discussed in [1], the European version of a flow gate approach is not the most efficient way of dealing with the scarce network resources. This is not about to change soon, but what is changing is how these capacities are then made available. Non-market based methods have largely been abolished and replaced by separate auctions per border. The auctions are organized by the TSOs and are typically for yearly, monthly and daily physical transmission rights.

Arbitrage between the various power exchanges is therefore already possible but explicit, requiring the purchase of physical transmission rights on a contract path. Besides being constrained by the available border capacities, arbitrage is also constrained by the time lag between the closing of the different border and power exchange auctions and the uncertainty that this brings, especially given the high price volatility. Several empirical

supporting measures, i.e. Nord Pool has a monopoly for cross-border trade on the internal borders of the Scandinavian countries and only generators that pass through Omel get a capacity payment. 
studies that compare the prices of border capacity with the price difference between exchanges indeed indicate that arbitrage is currently inefficient (see for instance [2]).

Market coupling ${ }^{5}$ solves this problem as it refers to the implicit auctioning of physical transmission rights via the hourly auctions organized by power exchanges one day ahead of delivery. Nord Pool (Elspot) already does this for several years for the total available capacity on the internal borders of the Scandinavian countries ${ }^{6}$. Since November 2006, the capacity available day-ahead ${ }^{7}$ on the internal borders of France, Belgium and the Netherlands that used to be auctioned in a separate market organized by the respective TSOs is now used by the exchanges to optimize the clearing of their day-ahead auctions. This so-called Trilateral Market Coupling (TLC) initiative is expected to be extended to include more countries ${ }^{8}$.

Market Coupling implies that exchanges can optimize the clearing of the offers and bids for electric energy submitted to their day-ahead auctions. As such, total gains from trade for the auction participants are increased. Often quoted benefits are also reduced price volatility and increased liquidity as orders can be matched across borders. Due to

\footnotetext{
${ }^{5}$ This terms can be confusing as it suggests that these markets were previously not coupled, which is not the case.

${ }^{6}$ Their implementation is commonly referred to as market splitting.

${ }^{7}$ Note that this is only a small fraction of total capacity, as most capacity goes to the yearly and monthly capacities which are still auctioned in a separate market.

${ }^{8}$ For instance a Memorandum of Understanding between exchanges, TSOs and regulators has been signed to include Germany.
} 
verticals in the aggregated order curves, the optimal solution can however be settled at different prices. In order for prices to give correct signals for network development and where to locate generation and consumption, price coordination between exchanges is necessary.

Section 2 introduces the market coupling optimization problem. Section 3 introduces the widely accepted approach to settle trade with network constraints, i.e. Location Marginal Pricing (LMP). Section 4 then illustrates that Locational Marginal Prices (LMPs) have important properties, but they are no always uniquely determined. Section 5 discusses price coordination between exchanges, including how this issue is being dealt with in the TLC initiative, how relevant it currently is and will be, when this initiative is extended to more countries.

\section{Market Coupling Optimization Problem}

The market coupling optimization problem involves demand and supply orders of different exchanges that need to be matched in order to maximize the total gains from trade $^{9}$. This means that the cheapest supply orders are matched with the most willing to pay demand orders. The only complexity in comparison with a single exchange optimization problem is that these orders come from different exchanges which represent a different network location. The demand and supply volumes traded on the different

\footnotetext{
${ }^{9}$ Resulting from demand that needs to pay less than its willingness to pay, supply that is paid more than what is wants to be paid and congestion rents that are transferred by the exchanges to the TSOs
} 
exchanges do not have to be equal, as long as the traded volumes equalize in total and the resulting flows between locations are feasible given the limited available network capacity.

For the market coupling optimization problem, the topology and capacities of the simplified network that need to be taken into account are given as they are predetermined by the involved TSOs. Given is also the volumes and prices of the orders that have been submitted. What needs to be determined is which orders are accepted at which hourly price for every exchange. The optimization problem can therefore be formulated as follows:

Maximize the value of demand minus the cost of supply:

$$
\underset{q}{\operatorname{Max}}\left(\sum_{z}\left(\sum_{j} q_{j z} P_{j z}-\sum_{i} q_{i z} P_{i z}\right)\right)
$$

With,

$P_{\mathrm{jz}}$ : price and limit of demand side order $\mathrm{j}$ submitted to exchange $\mathrm{z}$ (or introduced at location z)

$P_{i z}$ : price and limit of supply side order i submitted to exchange $\mathrm{z}$ (or introduced at location z)

$q_{i z}, q_{j z}$ : the decision variable representing the accepted volume of the respective orders

Subject to 
Order constraints, making sure that the accepted volume is not higher than the volume limit of an order:

$$
\begin{gathered}
q_{i z} \leq Q_{i z} \\
q_{j z} \leq Q_{j z}
\end{gathered}
$$

With

$Q_{j z}$ : volume limit of demand side order $\mathrm{j}$ submitted to exchange $\mathrm{z}$ (or introduced at location z)

$Q_{i z}$ : volume limit of supply side order i submitted to exchange z (or introduced at location z)

Network constraints ${ }^{10}$, respectively the constraints that equalizes the net injection at a location with what the off-takes at that location and the constraints that make sure that the flow on a interconnection of two locations is not higher than the capacity available between these locations:

$$
\begin{aligned}
& \forall z: \sum_{i} q_{i z}-\sum_{j} q_{j z}-\sum_{x} B_{z x}\left(\theta_{z}-\theta_{x}\right)=0 \\
& \forall z, x \in Z: B_{z x}\left(\theta_{z}-\theta_{x}\right) \leq \operatorname{Cap}_{z x}
\end{aligned}
$$

With

$B_{z x}$ : susceptance of the line interconnecting zone $\mathrm{z}$ and $\mathrm{x}$

\footnotetext{
${ }^{10}$ The constraints are DC load flow constraints, which are a simplification of the real power flow equations as for instance discussed in [8]
} 
$\theta_{z}$ : voltage angle

$\operatorname{Cap}_{z x}$ : capacity of the line interconnecting location $\mathrm{z}$ and $\mathrm{x}$

Note that in practice, the exchanges solve this optimization problem for every hour of the next day and the hours are dependent because of so-called block orders [5]. For reasons of clarity abstraction is made of block order in this paper.

\section{Price properties}

Locational Marginal Prices (LMPs) are the most obvious choice to settle the optimal solution to the market coupling optimization problem. It basically means that the orders of an exchange are settled at the price that corresponds to the shadow price of its market clearing constraint (4). LMPs have interesting properties. They for instance give efficient signals for network development and location of generation and consumption. Locational Marginal Pricing is also widely used; especially in the North American markets (see for instance [3]). Although a lot of literature is available discussing the properties of LMPs (see for instance [4]), much less is available on implementation issues of LMP. This paper discusses an implementation issue related to the verticals in the aggregated order curves of the exchanges that is relevant for the European context.

The properties of LMPs can be derived from the optimality conditions of the market coupling optimization problem (1)-(5), as has been done in [6] for the more generalized problem. This leads to the following equations that define the necessary relation between 
the LMPs and the shadow prices of (5), which correspond to the prices of the interconnections:

$$
\forall z, x: \sum_{x} B_{z x}\left[p_{z}-p_{x}+\mu_{z x}-\mu_{x z}\right]=0
$$

With

$\mathrm{p}_{\mathrm{z}}$ : the LMP, or simply locational price corresponding to location z. Note that demand and supply orders of a single location or exchange are cleared at the same price. $\mu_{x z}$ : the price of the line (or flow gate) interconnecting location $\mathrm{x}$ and $\mathrm{z}$, in the direction $\mathrm{x}-\mathrm{z}$, which corresponds to the shadow price of (5). Therefore this price is zero if constraint (5) is non-binding, which is the case when the line is not fully used.

Note that LMPs are not always as intuitive as one might think. Based on simplified examples in non-meshed networks, these prices have sometimes been attributed properties that the approach cannot deliver. Illustrative is what the authors in [6] call 'folk theorems' of LMP. Furthermore, in [7] prices observed in PJM (applies LMP) are discussed that at first sight can be considered abnormal but are actually normal LMP prices.

\section{Freedom in Prices}

\section{1. $\quad$ Price ranges}


Consider 3 exchanges PX1, PX2 and PX3 to which the orders listed in Table 1 are submitted. Figure 1 illustrates the implied aggregated order curves for the three exchanges separately and jointly. If the exchanges are not coupled they would have cleared a volume of respectively 100, 100 and $100 \mathrm{MWh}$ at a price of 10, 25 and 50 $€ / M W h$. Total gains from trade in that case would have been $18500 €^{11}$. If the exchanges would be coupled without binding network constraints, they would have cleared a total volume of $400 \mathrm{MWh}$ at a price of $25 € / \mathrm{MWh}$. In comparison with the non-coupled situation, total volume is the same in this illustration, but total gains from trade have gone up to $30500 €^{12}$. The difference, $12000 €$, is because at PX3 more demand can be supplied $^{13}$ and additionally the more expensive supply offer at PX3 can be replaced by the cheaper supply offer introduced at PX1 ${ }^{14}$.

The optimal solution implies a transfer of 200MWh from PX1 to PX3, i.e. an injection in the network of 200MWh at location 1 and a withdrawal of 200MWh at location 2. Figure 2 illustrates the possible locational prices and their corresponding export level. Note that these prices reflect the property of LMP that there is a single price per location to settle demand and supply at that location. Take for instance PX1:

- No supplier is offering at a price below 10€/MWh, while at such low prices demand will definitely want to be supplied fully, so that the corresponding import level for prices lower than $10 € / \mathrm{MWh}$ is $100 \mathrm{MWh}$

\footnotetext{
${ }^{11}$ (PX1:) 100MWh (90-10€/MWh) + (PX2:) 100MWh (90-25€/MWh) + (PX3:) 100MWh (90-50€/MWh)

${ }^{12}$ 300MWh (90-10€/MWh) + 100MWh (90-25€/MWh)

${ }^{13} 100 \mathrm{MWh}(90-10 € / \mathrm{MWh})$

${ }^{14} 100 \mathrm{MWh}(50-10 € / \mathrm{MWh})$
} 
- Demand does not want to pay more than 90€/MWh, while at such high prices supply will definitely want to be supplied fully, so that the corresponding export level for prices higher than 90€/MWh is 300MWh

- In between 10 and 90€/MWh demand wants to be fully supplied and suppliers want to supply all they offered as they can make a profit, so that the corresponding export level for prices between 10 and $90 € / \mathrm{MWh}$ is $200 \mathrm{MWh}$

- If the price is $10 € / \mathrm{MWh}$ or $90 € / \mathrm{MWh}$ respectively supply and demand can be curtailed as the orders are marginally accepted at those prices, so that there are respectively several corresponding import and export levels, as illustrated in Figure 2

In other words, an export of 200MWh corresponds to several possible locational prices at PX1. As illustrated in Figure 2, the same counts for PX3, which we will refer to as locational price ranges. Therefore the LMP property of having a single price per location alone does not fix the prices in this illustration. Another LMP property is that if there are no binding network constraints, the price of the lines should be zero. Figure 3 illustrates the impact on the network of the transfer between PX1 and PX3. Note that it is assumed that all line susceptances are equal so that 1/3 of the transfer goes via PX2 and 2/3 goes via the direct interconnection. Assuming that there is enough capacity to make this solution feasible, the remaining optimality conditions (6) translate into:

$$
\begin{aligned}
& 2 p_{1}-p_{2}-p_{3}=0 \\
& -p_{1}+2 p_{2}-p_{3}=0 \\
& -p_{1}-p_{2}+2 p_{3}=0
\end{aligned}
$$


These equations basically imply that the locational prices have to be equal. Given that the price of PX2 is fixed at 25€/MWh (Figure 2: there is no locational price range for PX2), this is the price for the three exchanges. In conclusion, an important LMP property is that LMPs are equal if there is no congestion in the network. Furthermore, in this example, there is only one set of prices that satisfies all LMP properties.

\subsection{Alternative sets of LMPs}

If we introduce binding network constraint to the example introduced in the previous section, the optimal solution changes. Figure 4 illustrates this with a binding capacity constraint between PX1 and PX3. In this network, a transfer between PX2 and PX3 is more interesting than a transfer between PX1 and PX3 as in the optimal solution without network constraints, because the latter uses more of the scarce network resource (double the amount) which offsets the supply cost advantage PX1 (10€/MWh) has over PX2 (25€/MWh). In this network setting, the optimal solution is to transfer as much as

possible between PX2 and PX3 and to use what remains on the interconnector between PX1 and PX3 for a transfer between these exchanges, as illustrated in Figure 4.

Figure 5 illustrates that the optimal solution yields two price ranges (PX2: $25<\mathrm{p}<90$; PX3: $50<\mathrm{p}<90$ ), but the export level of PX1 implies a price of 10. Given that there is a binding constraint between PX1 and PX3 so that $\mu_{13}$ is positive and given that $\mathrm{p}_{1}$ is 10 , (6) translates into:

$$
\begin{aligned}
& 20-p_{2}-p_{3}+\mu_{13}=0 \\
& -10+2 p_{2}-p_{3}=0
\end{aligned}
$$




$$
-10-p_{2}+2 p_{3}-\mu_{13}=0
$$

(10) - (12) is a set of 2 two linear independent equations with three unknowns ${ }^{15}$, meaning that there is some freedom in the prices. Indeed, solving the example in Matlab using the linprog solver yields prices of 10, 41 and 73€/MWh, respectively for PX1, PX2 and PX3 and solving it with CPLEX yields prices of 10, 30 50€/MWh. In other words, the example clearly illustrates that prices can differ significantly depending on which software is used to solve the problem. If no additional method is applied to consciously choose between the alternative sets of LMPs, the solution will depend on the solver software that is used.

\section{Price coordination}

\subsection{Importance of price coordination}

Perhaps the simplest way of dealing with price ranges is to allow every exchange to independently choose which price they take of the possible prices that correspond with the optimal export level that comes out of the market coupling problem. The consequence would however be that even the most basic LMP property, which is that prices should be equal if there is no congestion, is not necessarily satisfied. Even though the most willing to pay demand would still be matched with the cheapest suppliers, the distribution of gains from trade would be different. In this case, the network could generate congestion rents, giving incentives to further invest in the network, while increasing the network capacity would not improve welfare. In other words, only LMPs give correct signals for network development and also for where to locate generation or consumption. Therefore, 
the best way to coordinate prices is to use the shadow prices of the market clearing constraint, which are the LMPs.

The remaining question is what to do in case there are alternative sets of LMPs. Consider the illustration from the previous section. Table 3 summarizes some of the possibilities to choose from. As indicated in the table, the price of the line interconnecting PX1 and PX3 $\left(\mu_{13}\right)$ is always positive. However, the signal to invest can be double as high, depending on which LMPs are chosen. The highest $\mu_{13}$ value is actually the negative effect on total gains from trade if the capacity would be reduced with $1 \mathrm{MW}$, while the lowest $\mu_{13}$ value is the positive effect on total gains from trade if the capacity would be increased with 1 MW:

- 1 MW more, is 3/2 MWh more transfer between PX1 and PX3, which would mean replacing 3/2 MWh of supply in PX3 at 50€/MWh with supply from PX1 at $10 € / M W h$, which is a gain of $60 €(3 / 2(50-10))$

- 1 MW less, is 3/2 MWh less transfer between PX1 and PX3, which would reduce by 3/2 MWh demand in PX3 with a value 90€/MWh and supply in PX1 at $10 € /$ MWh which is a loss of $120 €(3 / 2(90-10))$

The signal you want to give for network development is of course the one corresponding to an increase of capacity, which in this case is the lowest value. For a convex optimization problem, it is in fact always the lowest value. It can therefore be concluded

\footnotetext{
${ }^{15}$ Actually bounded unknowns, as they are bounded by the price ranges
} 
that a good and straightforward way to choose between alternative sets of LMPs is to minimize congestion rents, subject to the LMP properties, such as equations (6)

\subsection{Relevance of price coordination}

Which price is chosen on a price range is of course only relevant if coupled exchanges are often faced with such price ranges and if they are significant. Figure 6 illustrates the price ranges on Belpex $^{16}$ for the first 2 months of operation. In $30 \%$ of the hours observed there is no price range, but in $20 \%$ of the hours there is a price range larger than 20€/MWh, with some observations peaking close to 400€/MWh. Given that a typical wholesale price is $50 € \mathrm{MWh}$, this is a very relevant part of the price formation on the power exchanges.

For the moment, the TLC initiative encompasses only France, Belgium and the Netherlands, which are aligned in that order. As the internal borders are not meshed, LMPs have more straightforward properties. For instance, the price of an interconnector is the difference between the location prices a both sides of the interconnector. Additionally the flow always goes from a high price region to the low price region, which is not necessarily the case if the network is meshed.

In [9], the price determination in case of price ranges is explained for TLC. The approach is specifically for three aligned markets. It is based on taking the middle price of an overlap between price ranges, subject to the LMP properties, which are called high level

\footnotetext{
${ }^{16}$ Note that these observations are from the first two months of operation of this exchange.
} 
properties of the algorithm. If market coupling is extended to more markets and meshed networks, the approach discussed in this paper could be used, which is to minimize congestion rents, subject to the optimality conditions in terms of prices of the market coupling problem.

\section{CONCLUSIONS}

Market coupling means that exchanges optimize the clearing of the electric energy orders submitted to their day-ahead auctions. In doing so, orders introduced at different locations are exchanged to the extent that the available network capacities allow. Prices at these optimal exchange levels can be undetermined on an interval or price range, due to the verticals in the aggregated order curves. For a single power exchange, a simple rule such as taking the middle price of the possible prices is sufficient. For coupled exchanges, coordination is however necessary in order not to distort the incentives for network development and locational incentives for new generation or consumption. Additionally it has been discussed that LMPs can be derived from the optimality conditions of the market coupling optimization problem, but that these conditions do not necessarily uniquely determine the prices, in which case it has been illustrated that the set of prices needs to be chosen that minimizes congestion revenues subject to the optimality conditions. 


\section{REFERENCES}

[1] Ehrenmann A. and Y. Smeers (2005). Inefficiencies in European congestion management proposals. Utilities policies, vol. 13 no. 2, pp135-152.

[2] Purchala K, Meeus L, Van Dommelen D. M, Belmans R. Usefulness of DC power flow for active power flow analysis. IEEE power engineering society general meeting 2005, San Francisco, California, USA.

[3] Ott A. L. Experience with PJM market operation, system design, and implementation. IEEE Transactions on Power Systems 2003; 18(2).

[4] Stoft S. (2002). Power system economics, designing markets for electricity. IEEE Press, 2002, 496 pp.

[5] Meeus L., "Power exchange auction trading platform design," PhD dissertation K.U.Leuven, ESAT-ELECTA, Advisors: R. Belmans \& S. Proost, ISBN 90-5682722-7, UDC 620.9, July 4, 2006; 152 pages, available at http://hdl.handle.net/1979/338.

[6] Wu F, Varaiya P, Spiller P. and Oren S. Folk theorems on transmission pricing: Proofs and counterexamples. Journal of Regulatory Economics 1996; 10: pp. 5-23.

[7] O’Neill R. P, Mead D. and Malvadkar P. On Market Clearing Prices Higher than the Highest Bid and Other Almost Paranormal Phenomena. The Electricity Journal 2005; 18(2): pp 19-27.

[8] Purchala K., Meeus L., Belmans R., "The analysis of the cross-border capacity allocation in the Benelux region”, Proc. 40th CIGRE Conference, 2004. 
[9] Belpex, Market Rules and Procedures, (Indirect) Participation Agreement, Appendix 2, TLC algorithm, 2006, available at: http://www.belpex.be/index.php?id=45 


\section{Figure Captions}

Figure 1: Aggregated order curves of three power exchanges separately and jointly ...... 21 Figure 2: locational price ranges corresponding to the optimal solution (Figure 1: intersection of aggregated order curves joined for the three exchanges) .......... 22 Figure 3: Impact optimal solution (Figure 1, intersection of aggregated order curves joined for the three exchanges) on the network ............................................. 23

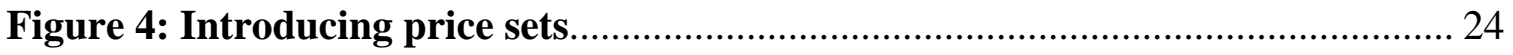

Figure 5: locational price ranges for solution in Figure 4 .................................... 25

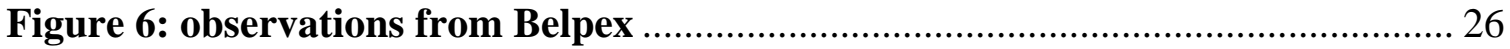




\section{Tables}

Table 1: Demand and supply orders introduced to PX 1 to 3

\begin{tabular}{|c|c|c|}
\hline PX1 & PX2 & PX3 \\
\hline \multicolumn{3}{|c|}{ Demand orders (bids) } \\
\hline 100MWh@ & 100MWh@ & 200MWh@ \\
90€/MWh & 90€/MWh & 90€/MWh \\
\hline \multicolumn{3}{|c|}{ Supply orders (offers) } \\
\hline 300MWh@ & 175MW@ & 100MWh@ \\
10€/MWh & 25€/MWh & 50€/MWh \\
\hline
\end{tabular}

Table 2: Demand and supply orders introduced to PX 1 to 3

\begin{tabular}{|c|c|c|c|c|}
\hline$(€ / M W h)$ & Linprog & CPLEX & Min CR & Max CR \\
\hline PX1 & 10 & 10 & 10 & 10 \\
\hline PX2 & 41 & 30 & 30 & 50 \\
\hline PX3 & 73 & 50 & 50 & 90 \\
\hline$\mu_{13}$ & 94 & 60 & 60 & 120 \\
\hline
\end{tabular}




\section{Figures}
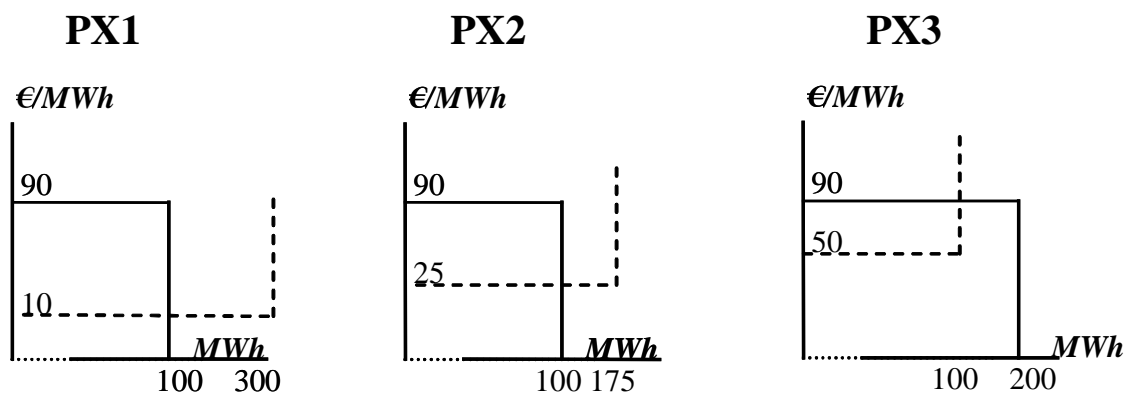

PX1 + PX2 + PX3

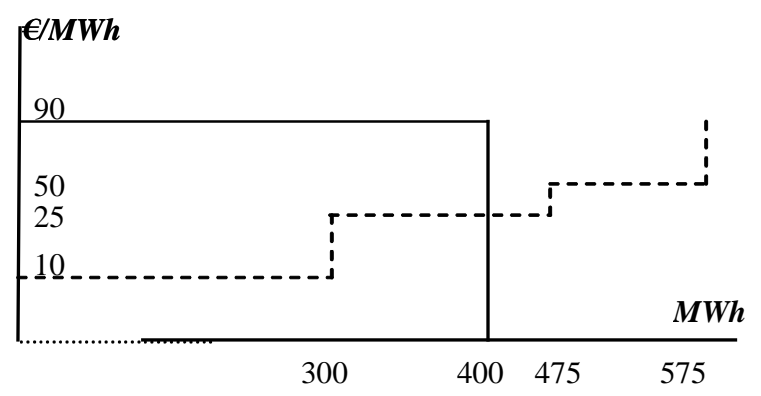

Figure 1: Aggregated order curves of three power exchanges separately and jointly 
PX aggregated order curves
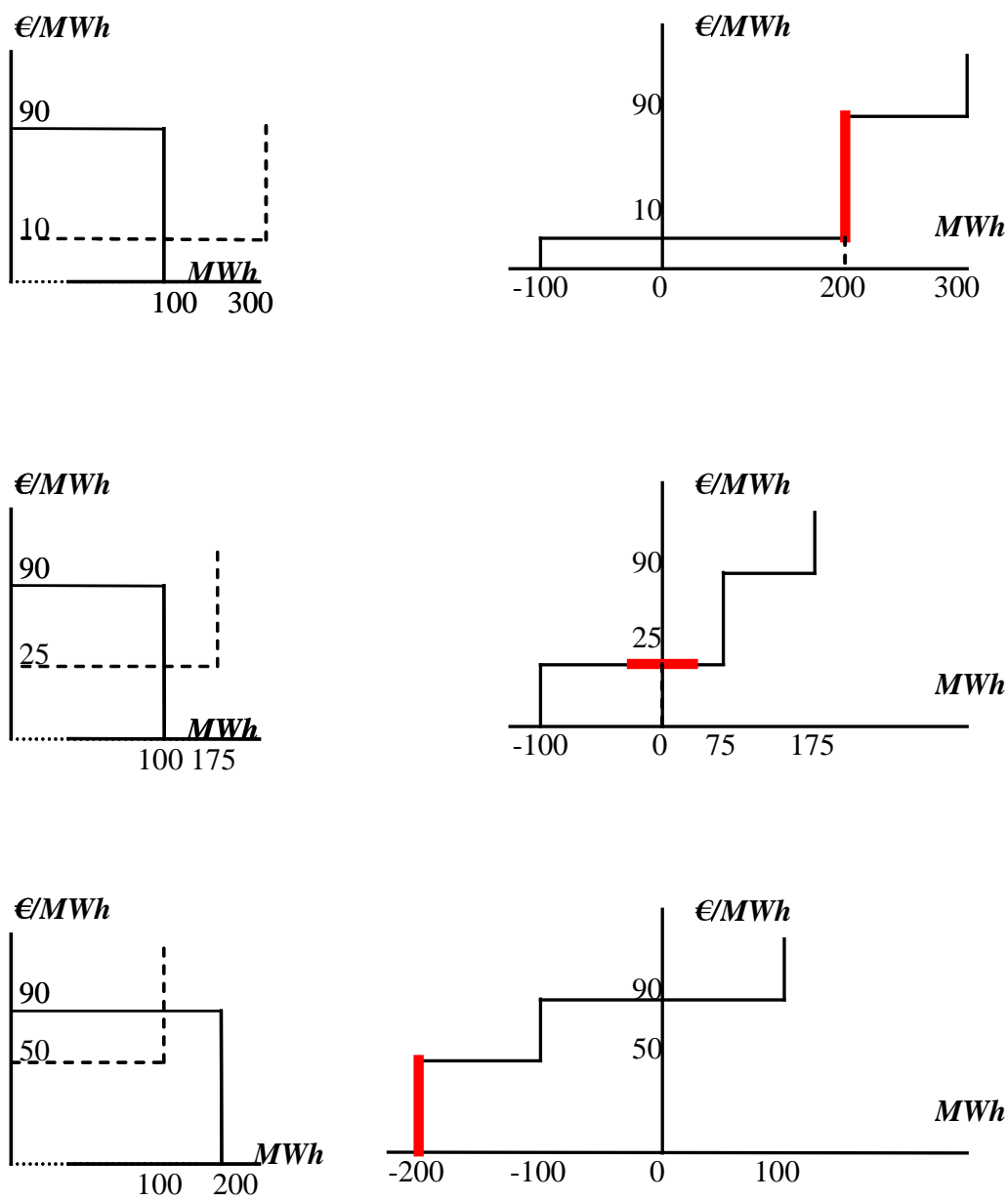

Figure 2: locational price ranges corresponding to the optimal solution (Figure 1: intersection of aggregated order curves joined for the three exchanges) 


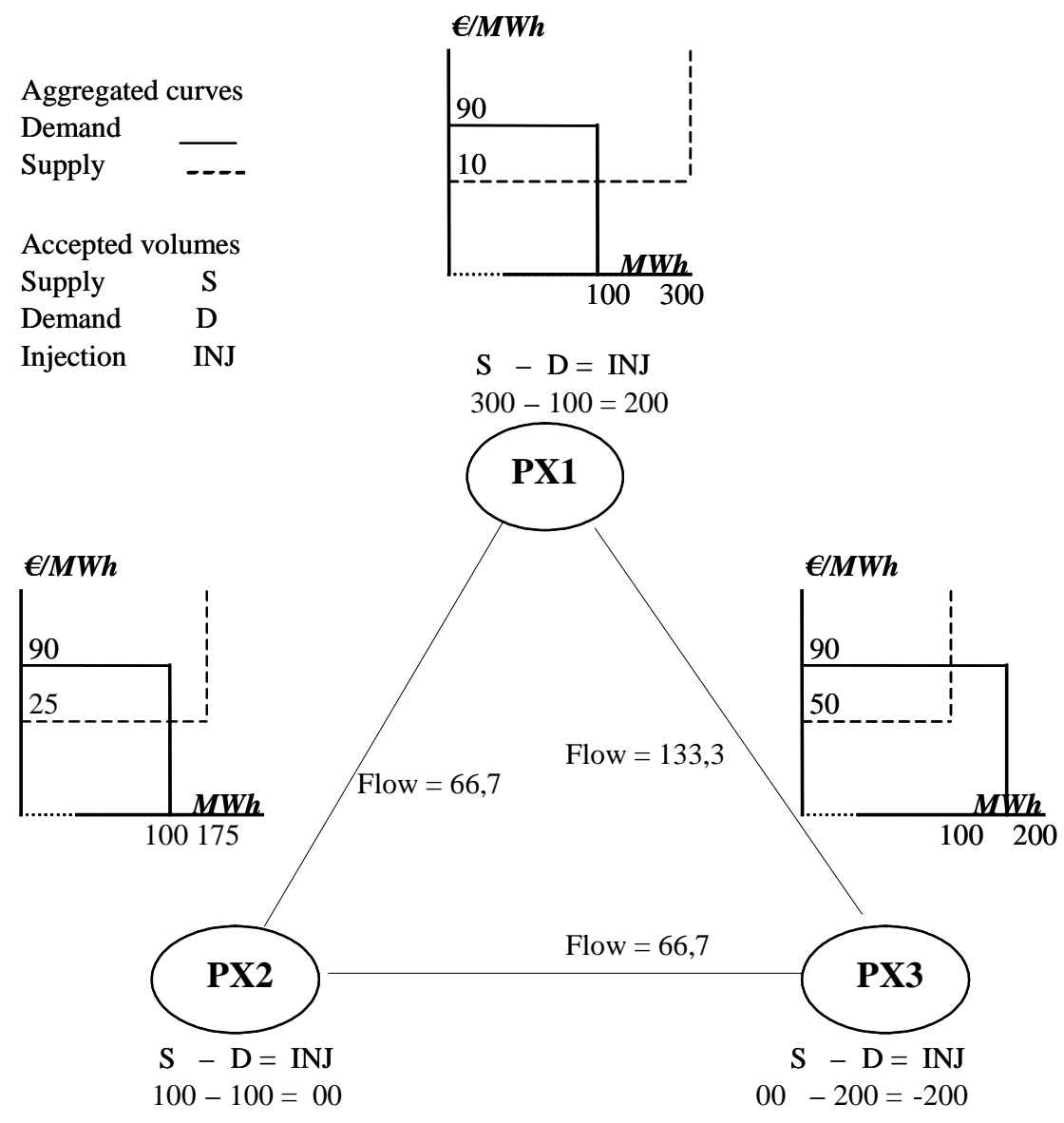

Figure 3: Impact optimal solution (Figure 1, intersection of aggregated order curves joined for the three exchanges) on the network 


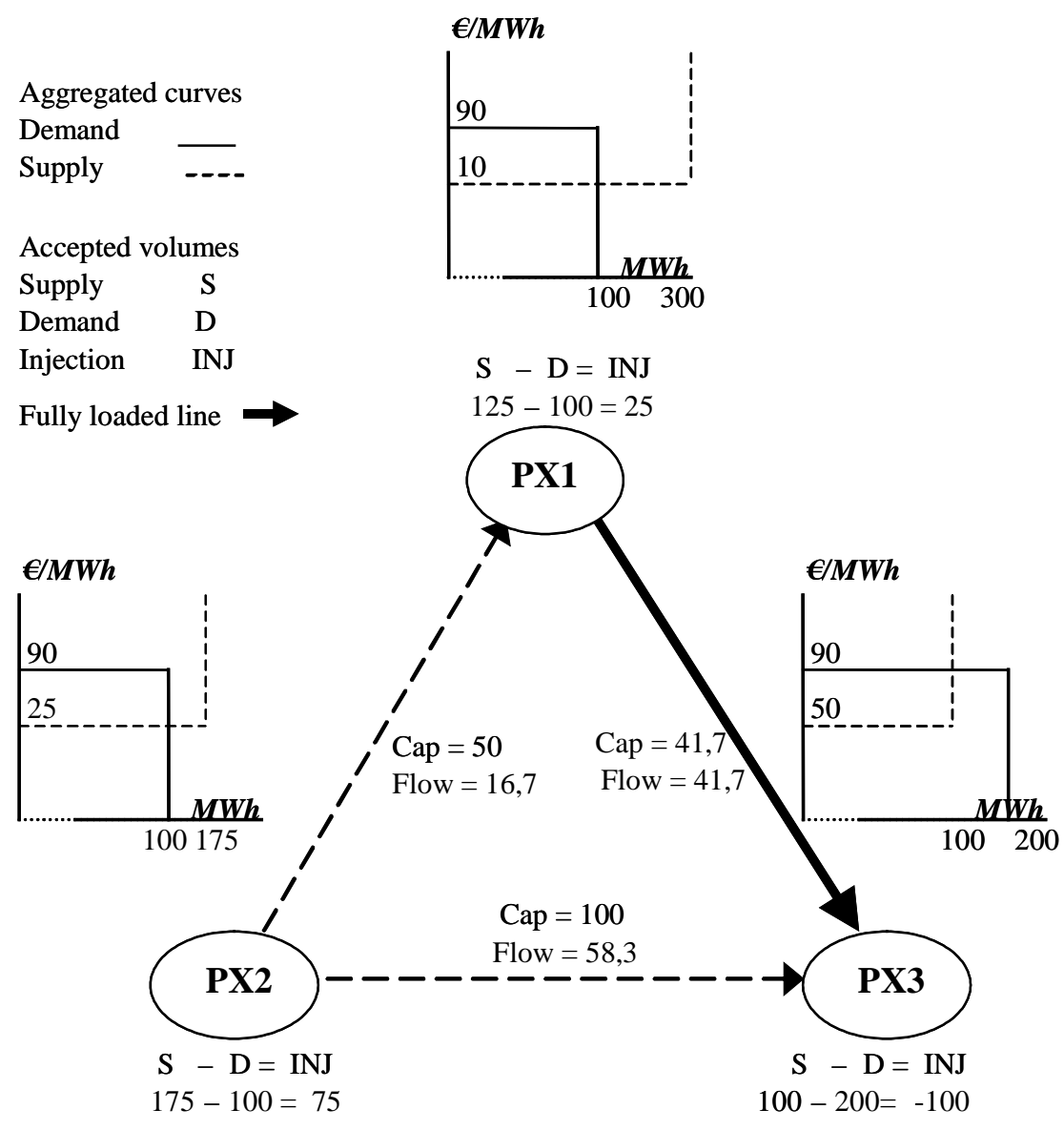

Figure 4: Introducing price sets 
PX aggregated order curves
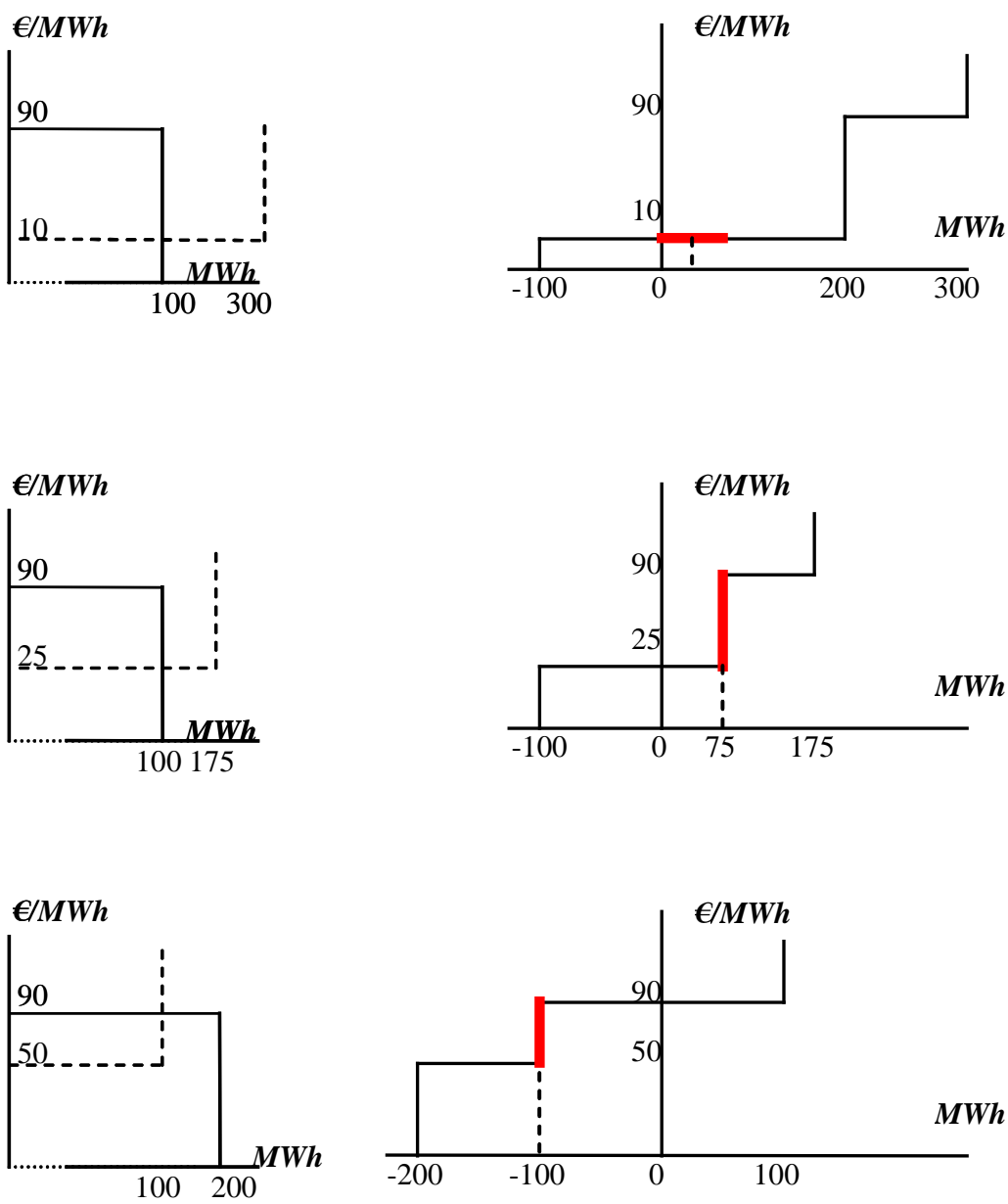

Figure 5: locational price ranges for solution in Figure 4 


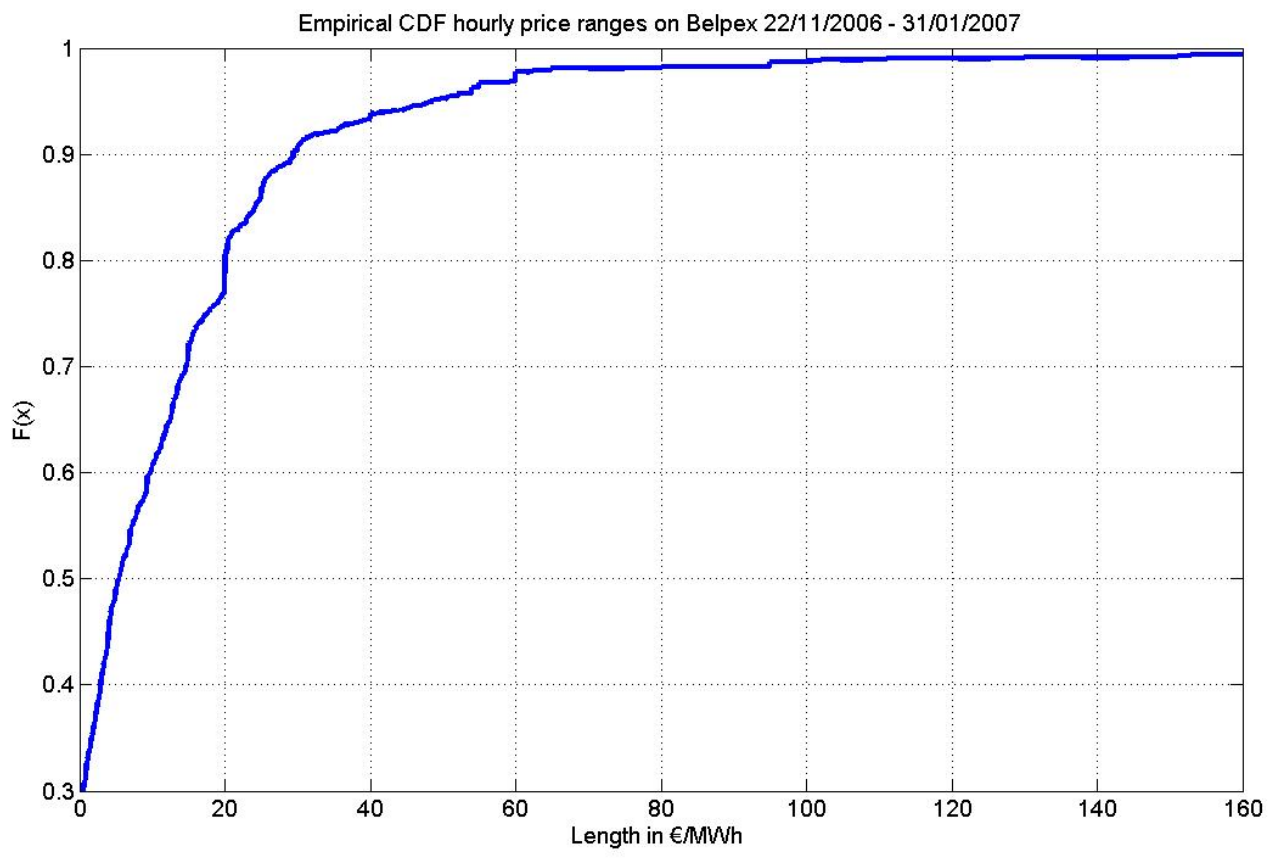

Figure 6: observations from Belpex 